\title{
Alternde Künstler als Liebhaber - Inspiration, (Pro-)Kreativität und Verfall: Anthonis van Dyck, Tizian und die Tradition der Renaissance
}

\author{
Ulrich Pfisterer
}

Die ,Alten Meister' scheinen den jungen Anthonis van Dyck auch zum Nachdenken über das Altern der Meister angeregt zu haben. ${ }^{1}$ Auf den rund 200 Seiten des Skizzenbuches seiner Italienreise (1621-1627) versammelte van Dyck nicht wie üblich zuvorderst Werke der Antike, sondern eine sehr persönliche Zusammenstellung von Kompositionselementen aus Gemälden der von ihm verehrten Maler Tizian, Raffael, Veronese und anderer, darunter auch Zeitgenossen wie Guercino oder Dirk van Baburen. Gegen Ende, auf fol. 109r-110v, finden sich dann drei Künstlerbildnisse von Tizian, Raffael und Sofonisba Anguissola. ${ }^{2}$ Eine ausführliche Beischrift erläutert allein zum letzten, mit knappen Strichen skizzierten Porträt dieser Reihe, der aus Cremona stammenden Sofonisba, dass van Dyck sie am 12. Juli 1624 als 96Jährige in Palermo angetroffen habe, beinahe erblindet, aber weiterhin von regem Geist und großem Interesse an der Malerei (Abb. 1).

Im aufgeschlagenen Skizzenbuch ihr gegenüber - auf der vorangehenden Seite war Raffaels Bildnis eines unbekannten jungen Mannes festgehalten, das van Dyck und seine Zeitgenossen offenbar für ein Selbstbildnis Raffaels hielten. ${ }^{3}$ Blättert man noch eine Seite zurück, sieht man sich schließlich einem alten Mann im Profil gegenüber, der zu einer jungen Frau aufblickt und ihr an den Bauch zu fassen scheint (die unterschiedliche Qualität der Zeichnungen im Skizzenbuch resultiert daraus, dass einige nachträglich von anderer Hand mit Feder nachgezogen wurden). Van Dyck notierte neben diese Szene: „mors Titiani“. Außer dieser Inschrift belegt auch die Tatsache, dass van Dyck nicht nur das ,Bildnis Raffaels“, sondern auch diese Zweiergruppe in seine ab 1645 im Druck publizierte Iconographie berühmter Männer aufnahm, dass van Dyck hier ebenfalls ein (Selbst-)Bildnis Tizians vor sich zu

\footnotetext{
${ }^{1}$ Zum Umgang mit, alt' und, neu ' im frühen 17. Jahrhundert vgl. nur Loh, 2007 und die Beiträge in Pfisterer \& Wimböck, 2011.

${ }^{2}$ Van Dyck, 1940, 70-73 (fol. 109r-110r); dazu Jaffé, 2001. Van Dyck zeichnete dabei nicht immer nach dem Original, sondern benutzte häufig Repliken oder Druckgraphiken als Vorlage.

${ }^{3} \mathrm{Vgl}$. van Dycks Identifizierung in der Iconographie, dazu Mauquoy-Hendrickx, 1991. - Zur Fortuna des Raffael-Porträt s. Wałek, 1991.
} 

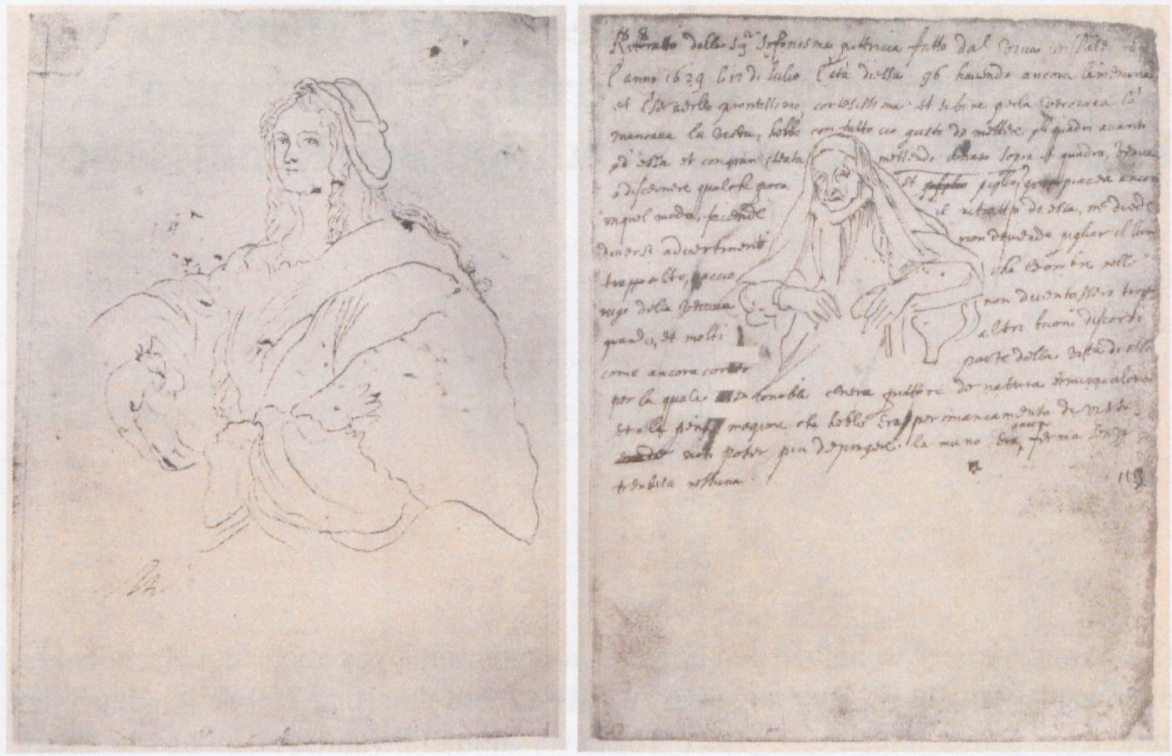

Abb. 1

haben glaubte (auf der Radierung ist nun zudem ein Totenschädel beigegeben). Wenig spätere Versionen dieser Darstellung ergänzen einen italienischen oder lateinischen Text, der die Identifizierung als ,Tizian mit seiner schwangeren Geliebten dann explizit macht (Abb. 2). ${ }^{4}$

Tatsächlich dürfte die Gesamtkomposition der Graphik (möglicherweise über heute verlorene Zwischenstufen vermittelt) auf ein Gemälde des Giovanni Cariani aus den Jahren um 1515/16 zurück gehen, das nicht Tizian, sondern einen unbekannten Greis mit Geldbeutel und eine junge Frau mit Kugel zeigt - ikonographisch eine Mischung aus dem zu dieser Zeit in Italien immer noch neuen Thema des ungleichen Paares und einer Schicksals-Allegorie. Allerdings weist der Greisen-Kopf Carianis auch (zufällig) eine erstaunliche Parallele zu einem jüngst aufgetauchten,

${ }^{4}$ „Ecco il Belveder! ô che felice sorte! / Che la fruttifera frutta in ventre porte / Ma ch'ella porte, ô me: vita et morte piano / Demonstra l'arte del magno Titiano.“ - „Ecce virgo quae grata suo est, nec pulchrior ulla: / Pignora coniugii ventre pudica gerit. / Sed tamen an vivens, an mortua, picta tabella / Haec magni Titiani, arte notanda refert." - Ein englischer Nachstich von Richard Gaywood ist dann betitelt: „Titian and His Mistress“; dagegen zielt eine deutsche Variante von Georg Walch wohl aus den 1640er/50er Jahren mit einem Vers aus Ovid, Amores 1, 9, 4 und einem gereimten deutschen ,Dialog' zwischen dem Greis und der jungen Frau nur mehr auf das Thema der ungleichen Liebe ab und nennt Tizian allein als inventor der Bildidee. - Vgl. dazu Wethey, 1971, 181f. (X-102); Chiari, 1982, 92f. (Kat. 75); Mauquoy-Hendrickx, 1991, Bd. 1, 116f.; und am umfassendsten Depauw \& Luijten, 1999, 240-248 (Kat. 32); Guderzo, M. In: Puppi, 2007, 361f. (Kat. 9). 


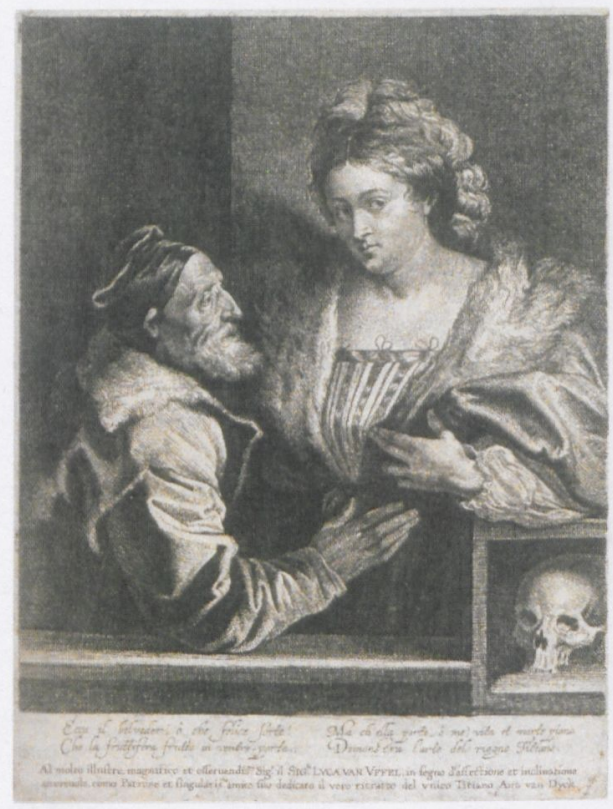

Abb. 2

gezeichneten Bildnis des alten Tizian aus den frühen 1560er Jahren auf, das möglicherweise sogar von dessen eigener Hand stammt. ${ }^{5}$

Wie kommt es zu dieser ungewöhnlichen Zusammenstellung dreier Künstlerbildnisse im letzten Teil des van Dyck'schen Skizzenbuches, wo ansonsten vor allem Kopfstudien und erotische Themen im weitesten Sinne versammelt sind? Ziel dieses Beitrags ist es zu zeigen, dass van Dycks Zeichnungen geradezu lehrbuchartig zentrale Ideen der Frühen Neuzeit über das Altern von Künstlern und Künstlerinnen und deren angeblich dadurch beeinflusste künstlerische Schöpferkraft vorführen. Dabei wurden die Vorstellungen zu Inspiration, Kreativität und künstlerischer Produktion während verschiedener Altersstufen stets in Parallele zu den unterschiedlichen Formen des Liebesverlangens und dem Vermögen zu biologischer Prokreativität gesehen. Van Dycks Zeichnung von ,Tizian mit seiner Geliebten ' und das eigentliche Vorbild Carianis spannen dabei den entscheidenden Zeithorizont auf, in dem sich diese Vorstellungen entwickelten - nämlich von den Jahren um 1500 bis ins frühe 17. Jahrhundert. Es geht in meinem Beitrag also nicht primär um die Darstellung alter Menschen und menschlicher Lebensstufen, nicht um ,Altersstile " und ,Alterswerke ' von Künstlern und nicht um Lebensalter-Zyklen der personifizierten Künste - alles insbesondere während der letzten Jahre schon einigermaßen gut un-

\footnotetext{
${ }^{5}$ Das Gemälde Carianis befindet sich heute in St. Petersburg, Eremitage; vgl. Pallucchini \& Rossi, 1983, 122 (Kat. 40); Fusenig, 1997, 76-80; Depauw \& Luijten, 1999, 246; Artemieva, I. In: Puppi, 2007, 362 (Kat. 10). - Zu dem gezeichneten Tizian-Bildnis, das engere Bezüge zu van Dycks Darstellung hat als das späte Selbstbildnis Tizians im Prado s. Rosand, 2009.
} 
tersuchte Themen. ${ }^{6}$ Vielmehr interessieren Bilder und Texte, in denen unterschiedliche Modelle zum Verständnis und zum Verhältnis von Lebenskraft, Liebeskraft und Schöpferkraft alternder Künstler und Künstlerinnen entwickelt werden.

\section{Lebenskraft und künstlerische Leistungskraft}

Drei ganz unterschiedlichen Vorstellungen, zu welcher Altersstufe die künstlerische Leistungskraft am höchsten sei, hatten sich bis ins frühe 17. Jahrhundert ausgebildet, wobei die Überlegungen zu den Künstlern häufig (und im Zeitalter des ut pictura poesis nicht überraschend) an denen zu Literaten und Dichtern orientiert waren.

Das Lob der Jugend und ,Frühbegabung (und damit implizit den Spott über den Verfall des Alters) sang vielleicht am eindrucksvollsten der bereits mit Anfang DreiBig verstorbene Girolamo Fontanella im Vorwort zum zweiten Buch seiner Ode (Bologna, 1633; 2. erw. Aufl. Neapel, 1638): Die Musen würden die Gesellschaft alter Männer verabscheuen, vielmehr wie verliebte Mädchen die Kurzweil mit Jünglingen genießen. Dem schnellen Lauf der Daphne könnten die Greise gar nicht mehr folgen; auch trage dann der alte Baum kaum noch Früchte. Wer jedenfalls eine große Menge ,Gedankenblitze“ vorzuweisen habe, dem müsse auch die Lebensenergie kraftvoll durch die Adern rinnen. Dagegen erstarre der göttliche furor poeticus mit dem Alter. ${ }^{7}$ Das , dichterische Feuer ${ }^{6}$ und die damit einhergehenden Tugenden so schon Cristoforo Giarda in seinen Icones Symbolicae (1628) - verlangten daher auch ewige Jugend für die Personifikation der Poesie: „Adspectus, crinis, os, vestis, caeteraque oblectationis instrumenta sunt animi plane adolescentis signa. Non enim musae senem amant, neque barbatis vatibus Apollo imberbis delectatur. Aetas senilis huic impar exercitationi, quae ardentiores spiritus desiderat, quae ingenium servens, sagax, acutum, ebulliens, plenum acrimoniae, poenae furens requirit. " 8 Ein Stammbuch-Blatt des Malers Johann Heintz von 1631 spitzt genau diesen Gedan-

${ }^{6}$ Vgl. nur die wichtigsten Veröffentlichungen dazu (je mit weiterer Literatur): Soussloff, 1987; Raupp, 1993a; Courtright, 1996; Campbell, 1998; Sohm, 2007. - Ein neuerer populärer Überblick von Dormandy, 2000; aus medizinischer Sicht etwa Rösler, Nesselhauf, Pfisterer et al., 2010.

${ }^{7}$ Fontanella, 1994, 109-111; das Vorwort zum ,zweiten Buch“ beginnt mit einem Verweis auf den Maler Apelles, der sich hinter seinen Gemälden versteckte, um die Kritik daran zu hören. Dann geht Fontanella auf den Vorwurf ein, seine Oden zu früh und schnell publiziert zu haben: „[... $]$ Le Muse, che sono figliuole della Memoria, abboriscono d'accompagnarsi coi vecchi, che sono padri della smemoraggine. [...] Le Vergini di Parnaso, come inamorate donzelle, più volentieri gradiscono la vaghezza de' giovani, che la severità degli attempati. Il vecchio, ch'è tardo nel moto e malagevole nel apsso, non può giungere frettoloso quella Dafne, che fuggendo dagli occhi d'Apollo e trasformandosi in allo, fu simbolo della gloria fuggitiva. Non è carrico di molte frutta quell'albero, ch'è carrico di molti anni. I furori poetici perdono la forza della divinità in un animo agghiacciato di senettù. Nella vecchiezza dell'inverno tengono silenzo gli uccelli; e nella primavera della gioventù cantano più soavamente i poeti. [...] Mostra copia di spiriti ne' concetti, chi raccoglie copia di spiriti nelle vene. E più purgato torna il suono delle sue rime, chi più purificati conserva gli organi del cervello. [...] .“-Zur Entstehung des Konzepts künstlerischer Frühbegabung s. Pfisterer, 2003.

${ }^{8}$ Giarda, 1628, 93. - Zu diesen Fragen auch Clement, 1960, 38f., der allerdings die Passage Giardas als Plädoyer für ein ,mittleres Alter' missversteht. 


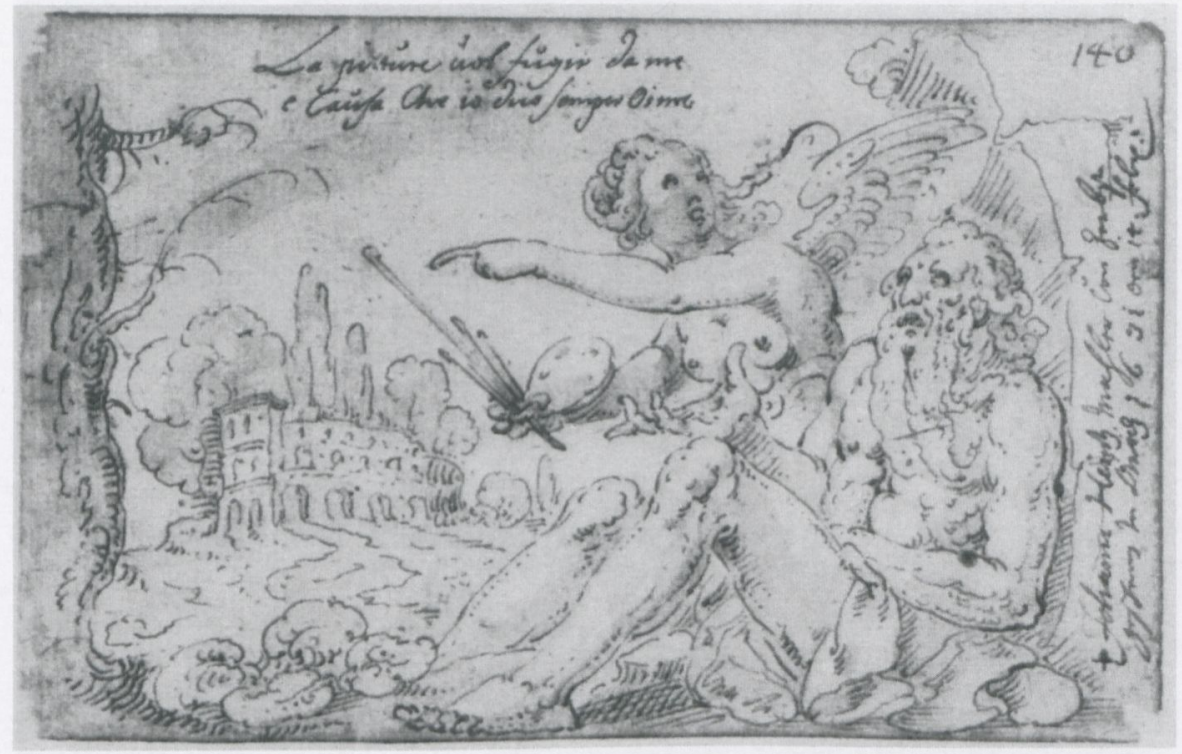

Abb. 3

ken auf das Thema Liebe zu (Abb. 3). ${ }^{9}$ Der sich in der Haltung eines ,Hiob im Elend, von seiner Frau verspottet " präsentierende Maler jammert in holprigem Italienisch: „Die Malerei will von mir fliehen / deshalb sage ich immer: Oh weh!“ Die im Gegensatz dazu jugendlich-erotische Nacktheit der Malerei-Personifikation deutet zwar einerseits auf ihr ehemaliges Liebesverhältnis zum Maler, andererseits auf die aktuell unerfüllten Bedürfnisse dieser Beziehung - wozu Karel van Mander 1604 kommentiert: „Die Malkunst gleicht einer schönen Frau, die sehr eifersüchtig über ihre Liebhaber und Anhänger wacht; denn wer sie nicht ernsthaft liebt und sucht, der erlangt sie nicht, und wer sie nicht ausübt und unterhält, den verläßt sie wieder." 10

Die Fragilität von Männlichkeit - Altersgebrechen, Impotenz und ähnliches rückt mit dem 16. Jahrhundert insgesamt verstärkt in den Fokus. ${ }^{11}$ Nachdem sich in den Jahrzehnten um 1500 die Vorstellung von den personifizierten Künsten und den Musen mit denen von Geliebten (und Modellen) der Künstler und Literaten (wieder) $\mathrm{zu}$ überlagern begonnen hatte, wurden nicht nur Liebesvermögen und künstlerisches Vermögen noch enger parallelisiert. Auch das Scheitern im einen Bereich konnte zum Signal des Scheiterns im anderen werden (wenn man nicht der ebenfalls zu dieser Zeit ausgebauten neuplatonischen Theorie der Sublimierung anhing). Jacopo de' Barbaris Tafel mit dem Doppelbildnis eines alternden Dichters und einer trauri-

\footnotetext{
${ }^{9}$ „La pitture uol fugir da me / e Causa Che io dico semper oime“. - Dazu und zur anderen Inschrift s. Raupp, 1993b.

${ }^{10}$ Van Mander 1604, fol. 143v; zitiert nach Raupp, 1993b.

${ }^{11}$ S. etwa Breitenberg, 1996; Long, 2002; Laam, 2006.
} 
gen Muse von 1502 scheint jedenfalls einer der frühesten, parodierenden Bildbelege für dieses Problem, das zur gleichen Zeit Konrad Celtis in seinen 1502 publizierten Amores thematisierte. ${ }^{12}$

Fontanellas abschließende Anspielungen auf den sich lichtenden Bart des Alters und die weißen Federn der Schwäne lassen besonders klar erkennen, gegen welches Argument und wen sich sein Vorwort richtet: gegen die Vertreter der Vorstellung, ein Schwan singe vor seinem Tod am schönsten - will sagen: das dichterische Können nehme mit dem Alter immer mehr zu. Auch diese Meinung vereinte im 16. und frühen 17. Jahrhundert eine große, vielleicht sogar die größte Schar von Anhängern hinter sich - wie etwa die populären Nachschlagewerke des Giovanni Pierio Valeriano (Hieroyglyphica, 1556) und dann Cesare Ripa (Iconologia, 1593/1603) zum Stichwort, Poesia“ belegen: „Il Cigno in vecchiezza continuamente va meglio articolando la voce, per estenuarsi la gola, \& così i Poeti continuamente vanno migliorando nell'arte loro con gli anni, come si racconta di Edipo Coloneo, \& di altri. "13 In diesem Sinne sind auch die positiv gemeinten Darstellungen älterer Dichter in Begleitung jugendlicher Musen seit dem frühen 16. Jahrhundert zu verstehen. ${ }^{14}$ Speziell auf Tizian wendet Pietro Aretino diese Vorstellung an, wenn er 1542 dem zu diesem Zeitpunkt möglicherweise erst knapp über 50jährigen Freund angesichts von dessen Porträt der kleinen Clarissa Strozzi attestiert: „Sicher ist, dass Euer Pinsel seine Wunder in der Reife des Alters bewahrt hat." 15 Noch expliziter reklamiert 1597 der Trevisaner Dichter und Maler Giulio Cornelio Graziani in seinem Epos Orlando santo für sich: „Die anderen Maler verlieren mit dem Alter / ihre Kunst, ich dagegen werde immer besser."16

Die Idee von einem mit zunehmendem Alter immer weiter entwickelten künstlerischen Können fügte sich nicht nur hervorragend in die Imitatio- und Idea-Lehren der Zeit ${ }^{17}$, sie ließ sich zudem verbinden mit dem antiken Sprichwort vom , kontinuierlichen Lernens bis ins Alter*. Diese Redewendung angeblich des Solon überliefert Platon und - ohne Herkunftsangabe-Seneca im 76. Brief an Lucilius: „Tam diu discendum est, quam diu nescias; si proverbio credimus, quam diu vivas. "In Italien wird diese Passage 1538 in einem Kupferstich des Agostino Veneziano unter der

\footnotetext{
${ }^{12}$ Ausführlich dargelegt bei Pfisterer, 2012.

${ }^{13}$ Pierio Valeriano, 1567, fol. 164r; Ripa, 1645, 493

${ }^{14}$ Vgl. die Beispiele bei Groos, 1998, etwa 302-304 zu einem Gemälde Carianis.

${ }^{15}$ Aretino, 1957-1960, Bd. 1, 217 (Brief Nr. CXLV): „Certo che il pennel vostro ha riserbati i suoi miracoli ne la maturità de la vecchiezza."

${ }^{16}$ Graziani, 1597, III, 33 - interessant auch die weitere Begründung als göttliches Geschenk: „Gli altri pittor ne la vecchiaia loro / mancon ne l'arte, et io fo meglio assai. / De importantia venendo alcun lavoro / trovo quel che non seppi trovar mai; / questo gran dono dal superno coro / credo che venga, e da i divini rai / acciò ch'io possa guadagnarmi il pane, / in questa lunga età, sera e dimane."

${ }^{17}$ Vgl. etwa aus den frühen 1530er Jahren Giulio Camillo Delminios L'idea dell'eloquenza (zitiert nach Bolzoni, 1984, 115): „Ma noi, che non abbiamo avuto né da giovanetti né da attempati documento certo e chiaro per il qual abbiamo possuto concepir questa idea, ci convien d'anno in anno perfino a la vecchiezza andarla concependo per gradi e rappresentarla or in un modo, or in un altro, perciocché non l'abbiamo ricevuta ad un tempo tutta."
} 


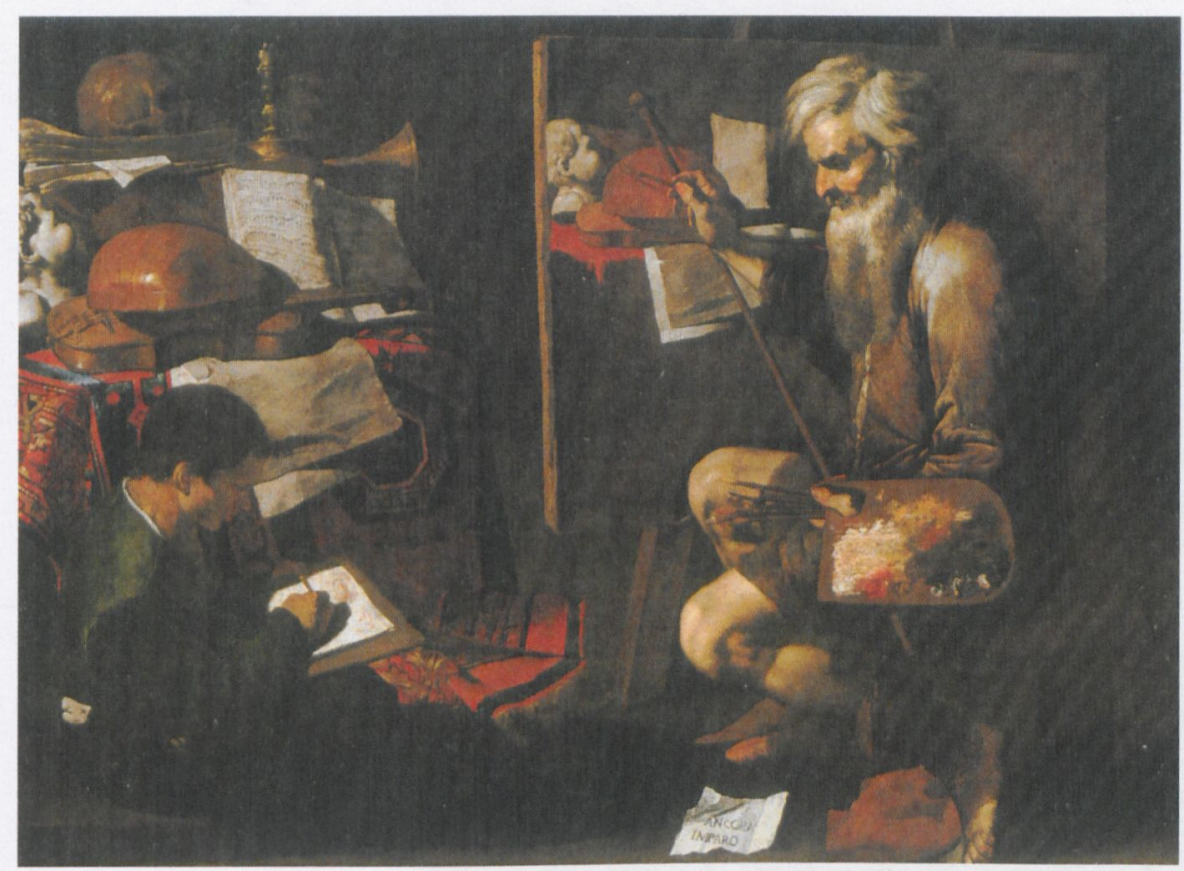

Abb. 4

Überschrift „ANCHORA INPARO“ illustriert, am unteren Rand ist Senecas Formulierung verkürzt zitiert und außerdem erläutert, dass ein Greis , zum zweiten Mal Kind“ sei („BIS PVERI SENES“): Zu sehen ist ein alter, gebeugter Mann, der sich mühsam mittels eines als Gehhilfe genutzten Wagens fortbewegt (eine freie Variante entsteht dann 1558 in Frankreich, wobei noch verdeutlichend ein kleines Kind bei seinen ersten Gehversuchen ergänzt wird). ${ }^{18}$ Die zunächst sehr positive Mahnung des dauernden Lernens konnte im 16. Jahrhundert dabei wiederum zugleich spöttisch als , Kindisch-Sein “ im Alter umgedeutet werden: Ausgerechnet von Sofonisba Anguissola stammt die Darstellung einer alten Frau, die bei ihren mühsamen Versuchen, Lesen zu lernen, von einem jungen Mädchen ausgelacht wird. ${ }^{19}$

Als Wahlspruch Michelangelos und dann in der Darstellung eines Maler-Ateliers aus den späten 1630er Jahren - auf einem cartellino zitiert - ist das „ANCORA

${ }^{18}$ Dazu Blake Smith, 1967, und Freedman, 1995, 111f., wo noch ein thematisch vergleichbares Emblem in Achille Bocchis Symbolicarum Quaestionum ... Libri V, Bologna 1574, symb. LXVII benannt ist. - Eine weiterer Nachstich nach Agostino Veneziano etwa in Freiburg, Augustinermuseum, Graph. Sammlung, in: Biegel, 1993, 33 und 295 (Kat. XI.22) (mit falscher Datierung ins späte 15. Jahrhundert).

${ }^{19} \mathrm{Zu}$ Anguissolas Zeichnung und einem Stich danach von Jacob Bos s. Gregori et al., 1994, 270 273 (Kat. 37f.). Ein zweites Beispiel für diese Ikonographie aus der Mitte des 17. Jahrhunderts, nun allerdings mit einem Knaben, der einen Greis unterrichtet und dabei einen Stock schwingt, von Pietro Paolini in englischem Privatbesitz, in: Tongiorgi Tomasi \& Tosi, 2009, 37 (falsch als „Lezione di Astronomia“" gedeutet). 
IMPARO“ aber jedenfalls positiv auf die Bildkünste zu beziehen (Abb. 4). Das Gemälde des Giovanni Dò in Neapel (möglicherweise auch als Selbstbildnis des Malers in der Rolle eines neuen Solon zu deuten) charakterisiert die Malkunst als lebenslange Herausforderung, die sich dem greisen Maler an der Leinwand immer noch genauso wie dem Knaben links bei seinen ersten Zeichenversuchen von Gesichtsteilen stellt. ${ }^{20}$

Die dritte Position schließlich beruft sich auf die ausgleichende Mitte. Vollendete Schaffenskraft sei weder mit unreifer Jugend noch zu hohem Alter vereinbar, verlange vielmehr die mittlere Lebensphase. Diese Position vertritt im Text etwa Raffaelo Borghini in seinem Riposo, 1584. ${ }^{21}$ Im Bild stellt eine neunteilige Folge der Lebensalter, die Nicolaes de Bruyn wohl im zweiten Viertel des 17. Jahrhunderts angeblich nach Entwürfen des Marten de Vos gestochen hat, den Höhepunkt der Kunst im 40. Lebensjahr dar (Abb. 5): Zu sehen ist Michelangelo bei der Arbeit an einer

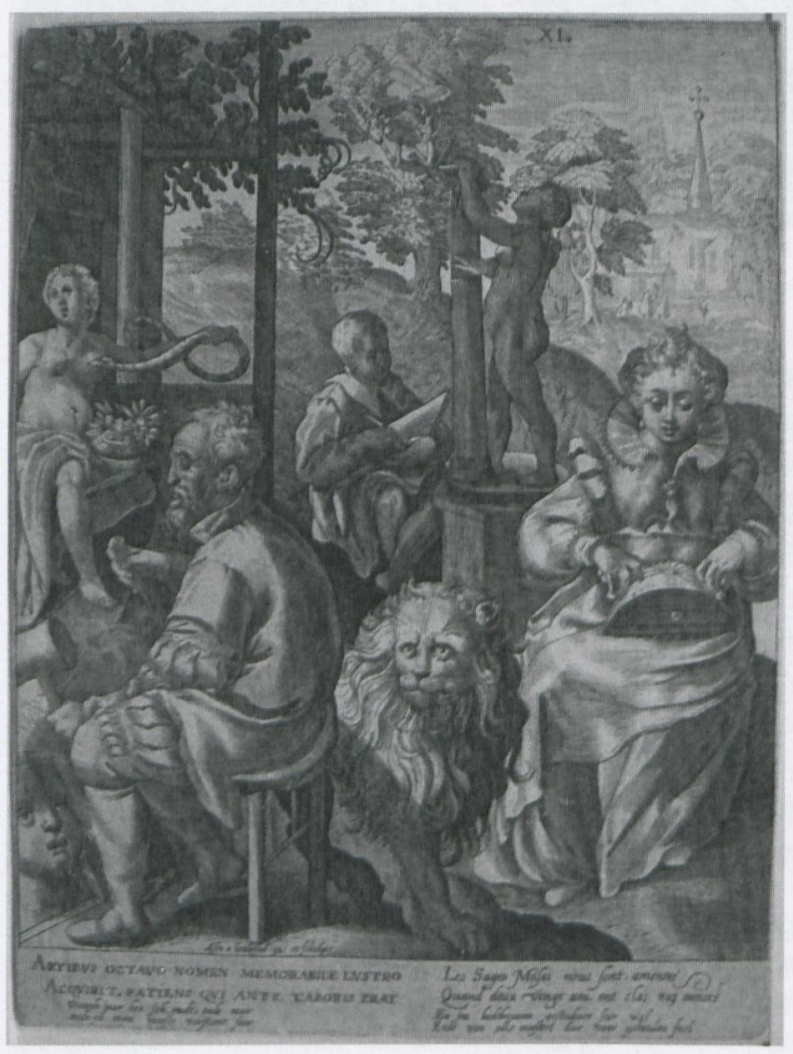

Abb. 5

${ }^{20} \mathrm{Zu}$ dem Gemälde liegt noch keine umfassende Deutung vor, vgl. bislang De Vito, 1996-1997, 31 und Farbtf. XIII; Spinosa, N. In: Seipel, 2001, 149 (Kat. I.31); Donati, 2008, 65; Schütze, 2010.

${ }^{21}$ Borghini, 1967, 196. 
Kleopatra-Statue, wobei ein ihn begleitender Löwe in der Tradition der Tiersymbolik in Verbindung mit den Lebensstufen des Menschen ${ }^{22}$ die höchste Schaffenskraft Michelangelos signalisiert. Daneben führt eine Frau das aus niederländischer Sicht weibliche Pendant zu dieser anspruchsvollen Kunst und mühevollen Arbeit aus: das Spitzenklöppeln. Ein Jüngling übt sich im Hintergrund im Abzeichnen einer Personifikation der ,Stärke', die erneut das 40. Lebensjahr als Akme des menschlichen Lebens kennzeichnet. Und die Inschrift betont nun unter anderem, dass die Musen den 40-Jährigen zugetan sind! ${ }^{23}$

\section{Die Biologie der Kreativität}

Kommen wir vor diesem Hintergrund zu van Dycks drei Künstlerbildnissen zurück und rufen uns in Erinnerung: Dass Tizian und Raffael als zwei der Heroen van Dycks dargestellt sind, bedarf keiner weiteren Erklärung, wohl aber die Auswahl gerade dieser beiden apokryphen Porträts, die vermeintlich den einen als greisen Liebhaber, den anderen als schönen Jüngling zeigen. Sofonisba dürfte dagegen zunächst als eine Art ,Kuriosität' aufgenommen sein - so wie sie ihre Karriere seinerzeit als Wunder-Kind begonnen hatte, so erscheint sie nun als Wunder-Greisin, als letzte, uralte Augenzeugin aus der Zeit der großen Meister der Renaissance.

Im Gegenüber mit Raffael ergeben sich aber noch weitere Assoziationen: Raffael ist der Frühverstorbene, der in kürzester Zeit alles erreicht und den die Natur aus Neid darüber, dass seine Gestalten schöner aussehen als die ihren, vorzeitig (eigentlich: mit 36 Jahren) ins Grab ruft - so erklärt jedenfalls seine weithin bekannte Grabinschrift (zu erwähnen ist auch die zweite Überlieferung, wonach Raffael an Erschöpfung beim Liebesakt starb) ${ }^{24}$ Vermutlich benutzt van Dyck das Modell Raffaels auch für ein Selbstbildnis aus diesen Jahren - allerdings konnte ein wenig späteres Bildnis van Dycks bereits zu Beginn der 1630er Jahre auch in Verbindung zu Tizians Altersporträt gesetzt werden. ${ }^{25}$

Sofonisba dagegen verbraucht ihre Lebenskraft während ihres langen Malerinnen-Daseins vollkommen. Van Dyck - oder zumindest seine italienischen Zeitgenossen - dürften dabei auch geschlechtsspezifische Unterschiede assoziiert haben:

\footnotetext{
${ }^{22}$ Wanders, 1983.

${ }^{23}$ Hollstein, 1951, 23 (Kat. 201-209); die Personifikation der Stärke rekurriert auf eine Statuette des Conrat Meit, vgl. Burk, J.L. In: Eikelmann, 2006, 84-87.

${ }^{24}$ Vgl. nur Lomazzo, 1973, Bd. 1, 250 zu Raffael: ,in cosí poco tempo fece quello che alcun’altro, nel corso di molti e molti anni, non aveva fatto giamai, come che fosse però universale in tutte le altre parti, sí che di anni trentasei finí la vita, giunto a sí alto segno, che a piú sublime non poteva poggiare." - Zur Analyse vorläufig Verrier, 2003.

${ }^{25}$ Vgl. etwa Barnes, S.J. In: Wheelock, 1990, 167-169 (Kat. 33): hier nicht erkannt, dass das Raffael-Porträt als Selbstbildnis angesehen wurde. - In einer Folge von radierten Studienblättern des Michiel Snyders, die nur aus den allerersten 1630er Jahren datieren kann, erscheint van Dycks um 1630 entstandenes Bildnis, das dann auch für die Iconographie verwendet wurde, neben dem Tizian-Kopf aus ,Tizian und seine Geliebte ' und einer Platon-Büste; vgl. Depauw \& Luijten, 1999, 295.
} 
Vasari hatte just am Beispiel Sofonisbas den für ihn verwunderlichen Umstand, dass eine Frau so gut malen könne, damit erläutert, dass sie ja auch Kinder zu gebären im Stande sei, ihre quasi biologisch gegebene Fähigkeit zur ,Abbildung ' und Reproduktion daher weit weniger Bewunderung verdiene als die ihrer Kollegen, die sich diese Fähigkeit auf rein geistiger Ebene anzueignen hätten. ${ }^{26}$ Da Sofonisba trotz mehrerer Ehen keine biologischen Kinder zur Welt brachte, scheint bei ihr die Alternative besonders deutlich: Ihre Werke sind ihre Kinder. Ohne die Tradition der Vorstellung von den Werk-Kindern seit Platon hier eingehender verfolgen zu können, sei nur darauf verwiesen, dass schon um 1500 Frauen ihre körperliche ,Sterilität' mit ihren Geistes-Produkten zu rechtfertigen versuchten. ${ }^{27}$

Vor allem aber wurde auch zwischen der , alternden Praxis“ und der ,ewig jungen Theorie " unterschieden. Giovan Paolo Lomazzo erklärte in seiner Idea del tempio della pittura (1590), dass die allein mit der Praxis vertrauten Maler mit dem Altern und Verfall ihrer Körperkräfte auch ihr Vermögen zu Malen verlieren würden, wogegen die auch theoretisch versierten Maler die „Schönheit ihres Geistes und die Schärfte ihrer Urteilskraft" behielten und damit auch ihre Schaffenskraft. ${ }^{28}$ Fulvio Mariotelli entwarf für Ripas berühmte Iconologia (seit der Ausgabe 1618) Personifikationen von, Theorie ' und ,Praxis', die diese Vorstellung durch den anschaulichen Alters-Kontrast von Frauen weiter ausbauten: Den Höhenflug der Theoriebildung, die vom Allgemeinen und den ewigen Gesetzen ausgeht, versinnbildlicht eine junge Frau mit Zirkel an der Stirn und nach oben gerichtetem Blick. Dagegen erscheint die Prattica als Greisin, die mit ihrem Zirkel nicht nur am Boden und also ausgehend von Einzelfällen arbeitet, sondern diesen auch als eine Art Stock benutzt. ${ }^{29}$ In Pietro Testas in den 1630er Jahren konzipiertem Liceo della Pittura ist die Praxis dann radikal als blinde Alte dargestellt. ${ }^{30}$ Mariotellis (bzw. ,Ripas ') und Testas Personifikationen sind zwar qua grammatischem Geschlecht beide weiblich. Auf konkrete Personen übertragen, musste insbesondere zu Zeiten van Dycks aber der jugendliche Raffael als der Theorie-Künstler der Renaissance schlechthin gelten durch seinen sprichwörtlichen Hinweis auf die „,certa idea“, nach der er seine Gestalten bilde. ${ }^{31}$ Die weitgehend erblindende Sofonisba dagegen wäre als ,Praktikerin ' deutlich abqualifiziert, so lobend van Dycks Stichworte zu ihr auf den ersten Blick auch scheinen mögen.

Bezieht man in diese Überlegungen nun das Bildnis Tizians mit seiner Geliebten ein, werden auch hier die zugrunde liegenden Vorstellungen einer ,Biologie der

\footnotetext{
${ }^{26}$ Mit unterschiedlichen Deutungen Jacobs, 1994 und nochmals Jacobs, 1997; anders dagegen Christadler, 2000. - Zu Gender-Aspekten des Alterns allgemein etwa Filipczaj, 1997, 22f. und Bake, 2005.

${ }^{27}$ Ein Beispiel bei Ahmed, 2008.

${ }^{28}$ Lomazzo, 1973, Bd. 1, 252: „non però possano mai perdere la bellezza dello spirito e la sottigliezza del giudicio che serve all'arte et alla prattica sottile regolata dalla teoria."- Dazu und zum Folgenden ausführlich Campbell, 1998, 34-71.

${ }^{29}$ Ripa, 1645, 496 und 622f.; vgl. Cropper, 1971; Garrard, 1986, 356-359; Schuster Cordone, 2009, 158-161.

${ }^{30}$ Dazu Cropper, 1971, und Campbell, 1998.

${ }^{31}$ Vgl. nur Winner, 1992, und Perini, 2000.
} 
Kreativität' ganz offensichtlich. Zunächst ist zu betonen, dass in Wirklichkeit auch der greise Tizian mit nachlassender Seh-, Körper- und Schaffenskraft zu kämpfen hatte, wie einige ,inoffizielle ' Zeugnisse überliefern. ${ }^{32}$ Dagegen setzte Tizian aber als einer der ersten neuzeitlichen Künstler eine intensive Selbststilisierung seines Alters und, Alterswerks ${ }^{6}{ }^{33}$ Und die frühen gedruckten Biographien Tizians folgen dem allesamt, indem sie von seiner ungebrochenen Schaffenskraft bis ins hohe Alter berichten. Für Informationen zu Tizian - die insbesondere auch die enigmatische Randnotiz „mors Titiani“ erklären helfen könnten - hätten van Dyck theoretisch zur Verfügung gestanden die italienisch verfasste Vita Giorgio Vasaris (1550 bzw. eher die zweite Ausg. 1568), Ludovico Dolces L'Aretino (1557), Raffaelo Borghinis Il Riposo (1584), die niederländische Lebensbeschreibung des Karel van Mander (1604, zweite Aufl. 1618), ein ganz aktuell 1622 anonym publiziertes Breve Compendio della vita del famoso Titiano und die bislang in der Forschung kaum beachtete Weltchronik des Pieter van Opmeer und Laurens Beyerlinck von $1611 .{ }^{34}$ Allein diese letzte konzentriert ihren kurzen Eintrag zu Tizian vor allem auf die Themen Alter und Tod (bzw. Überwindung des Todes durch die Kunst), so dass die Vermutung nahe liegt, van Dycks Informationen über Tizian seien möglicherweise gerade durch diese Publikation mitbestimmt gewesen. ${ }^{35}$

Dagegen wissen die frühen gedruckten Viten Tizians nichts davon, dass sich der alternden Maler als besonders aktiver Liebhaber betätigt hätte - und von der ihm tatsächlich um sein 58. Lebensjahr geborenen, illegitimen Tochter dürfte man zu Zeiten van Dycks ebenfalls nichts mehr gewusst haben. ${ }^{36}$ Gleiches gilt für den Bericht des Ferrareser Botschafters bereits vom Ende des Jahres 1521, wonach sich der zu diesem Zeitpunkt freilich in seinen besten Mannesjahren stehende Tizian mit seinen Modellen teils exzessiv verausgabte: „Ich habe Tizian gesehen, der überhaupt kein Fieber hat. Er sieht gut aus, wenn auch etwas erschöpft; ich vermute, dass die

${ }^{32}$ Am deutlichsten äußert sich der Kunsthändler Niccolò Stoppio in einem Brief vom Februar 1568, vgl. Hope, 1980, 151 (vgl. auch 161); die nachlassenden Fähigkeiten Tizians sind dann auch mehrfach Thema in den Korrespondenzen des habsburgischen Hofes, s. Mancini, 1998, etwa 405f. (Nr. 285f.) und 411 (Nr. 291).

${ }^{33} \mathrm{Vgl}$. Fletcher, 2003, und vor allem Sohm, 2007.

${ }^{34} \mathrm{Vgl}$. vorläufig - etwa ohne Verweis auf Opmeer/Beryerlinck - die Zusammenstellung bei Hope, 1993; Van Mander, 1604, fol. 177v berichtet in den Norden: „[... ] want sulcke leste zijn wercken verminderden zijn vermaertheyt oft gerucht. Hy was t'zijnen 86. Jaer noch ghevonden met den pinneelen in de handt / en sitten werckende.“; 1622 heißt es etwa über Tizians Lebensende in einem Halbsatz bei Tizianello, 2009, 58: ,ridotto agli ultimi anni della sua vecchiezza, imitando il buon Socrate, soleva dire che, se l'occhio lo servisse, allora gli avrebbe dato l'animo di cominciare qualche opera degna; [...]." - Zur Rezeption Tizians auch Loh, 2007, etwa 101f.; für den englischen Horizont van Dycks, s. Reinhardt, 1999.

35 Opmeerus \& Beyerlinck, 1611, Bd. 2, 40: „Quid vero naturae ars sociata valeat, stupendis in omne aevum operibus docuit TITIANVS VCCELLO DE CADOR Reipub. Venetae Pictor illustris, ut Laudis Apelleae metuit sua sidera fulgor / Sospite quo vinci, quo moriente mori. Aetatem, quod rarum in Pictoribus, ad XCVI. annum modesto cultu, \& paterno contentus salino, cibi etiam potusque parsimonia extraxit; ita ut non prius esse desineret, quam cum naturae debilitatus humor quotidiana dispendia reparare non valeret. Mortuus est anno M.D.LXXVI.“- Das Gedicht übernommen aus dem Leonardo-Stich in Reusner, 1589, R.2 [276].

${ }^{36}$ Zur späten Tochter Brunetti, 1935. 
Mädchen, die er öfters in verschiedenen Posen malt, bei ihm Wünsche wecken, die er dann mehr befriedigt, als es seine begrenzten Kräfte erlauben. “37

Es scheint vielmehr die dichte, bis ins Spätwerk nicht abreißende Reihe von Darstellungen erotisch-(halb)nackter Frauen, aus denen auf die ,Wirklichkeit ' des Künstlers rückgeschlossen wurde. Es sind genau diese Themen, die auch van Dyck in seinem Skizzenbuch besonders interessierten. Und es ist diese Verbindung von Künstler, Liebe und weiblicher Schönheit, die Tizian selbst etwa in der GemäldeSerie der Orgelspieler mit Venus und möglicherweise in einem heute nur durch Kopien überlieferten Selbstbildnis mit Venus-Statuette intensiv und selbstreflexiv für sich und seine Kunst thematisiert hat. ${ }^{38}$ Es geht bei diesen Bildern wie dann bei Van Dycks Tizian-Porträt also nicht um wirkliche oder vermeintliche Geliebte des Malers - und deshalb schien seinem Erfinder das Bildnis Tizians mit junger Geliebten auch nicht in Gefahr, mit den vielen negativ konnotierten, spottenden und mahnenden Darstellungen ungleicher Paaren verwechselt zu werden (was dann allerdings doch spätestens bei einem deutschen Nachstich von Georg Walch der Fall war). Vielmehr sollte das Sinnbild des alten Liebhabers Tizian, der mit seiner jungen Geliebten Kinder zeugt, auf Tizians kreative Potenz selbst im Alter verweisen, auf seine Liebe zu Malerei und Werken und auf die ungebrochene Bilderflut aus seiner Produktion.

Die Selbststilisierung Tizians als Liebeskünstler, wie sie das vermeintliche Selbstbildnis mit Geliebter aufgreift, scheint auch in den Schriftquellen ab der zweiten Hälfe des 16. Jahrhunderts eine sehr intensive Rezeption erfahren zu haben, am deutlichsten vielleicht bei Antonio Persio 1576, der Tizians Konzentration und Versenkung in den Schaffensprozess explizit als eine Art Liebesakt beschreibt, aus dem das ,Werk-Kind“ hervorgeht: ,[il] gran Titiano padre del colorire, il quale secondo ho udito di sua bocca, et di quegli che sono ritrovati presenti a' suoi lavori, quando volea disegnare o colorir alcuna figura, tenendo avanti una donna o un huomo naturale, cotal oggetto così movea la vista corporale di lui, et il suo spirito così penetrava nell'oggetto di chi ritirava, che facendo vista di non sentire altra cosa, che quella, veniva a parere a' circostanti d'esser andato in ispirito, dalla quale astrattione si cagionava che egli nell'opra sua riuscisse poco men che un'altra natura, tanto bene esprimendo la carnatura et fattezze d'essa. Così dunque avenir si estimerà della donna et dell'huomo che s'amano infra di loro [... . "39 Bei Sperone Speroni finden sich bereits etwas früher ähnliche, wenngleich noch nicht ganz so

\footnotetext{
${ }^{37}$ Hope, 1980, 57f.; die Übersetzung in Anlehnung an Borggrefe, 2006.

${ }^{38} \mathrm{Ob}$ es sich wirklich um die Kopie eines Selbstbildnisses handelt oder nicht doch eher um ein Tizian-Pasticcio des Pietro Della Vecchia aus der Mitte des 17. Jahrhunderts kann hier nicht diskutiert werden, auch nur als Pasticcio würde das Bild die hier vertretene Deutung unterstützen, s. zuletzt Dal Pozzolo, E.M. In: Puppi 2007, 360f. (Kat. 8) und Artemieva, 2008; zur OrgelspielerSerie und dem größeren Kontext etwa Pardo, 1993, und Suthor, 2004.

${ }^{39}$ Persio, 1999, 69f. Auf diese Stelle verweisen etwa bereits Hope, 1980, 169f. und Emison, 2004, 163. - Möglicherweise handelt es sich bei diesem Gedanke um eine Variante der Idea-Lehre und des Maler-Beispiels von Zeuxis und den Jungfrauen, vgl. etwa wenig zuvor Partenio, 1565, II, 66: "Quemadmodum enim in pingendo, sculpendove ita in eloquentia ideam quondam animo proponemus, ad cuius exemplar quicquid fingamus, aut dicimus, conferamus. Quod praeclare Zeusis in
} 
explizite Vorstellungen von der Liebe Tizians zu seinen Gemälden. ${ }^{40}$ Näher an van Dyck wird dann etwa Giulio Cesare Gigli in seiner 1615 gedruckten La Pittura trionfante das Gefolge der Maler hinter dem Triumphwagen der Malerei, angeführt von den Venezianern, darunter Tizian, als Versammlung ihrer Liebhaber beschreiben und intensiv die Metaphorik der Kunst-Liebe aufrufen. ${ }^{41}$ Van Dycks Wahl eines Bildnisses Tizians am Rande des Todes mit der schwangeren Geliebten - und unter Missachtung aller anderen (authentischen) Tizian-Bildnisse - lässt sich allein in diesem Deutungshorizont verstehen.

\section{Zusammenfassung und Ausblick}

In Anthonis van Dycks Skizzenbuch seiner italienischen Reise (1621-1627) findet sich gegen Ende eine bemerkenswerte Zusammenstellung von drei Künstlerbildnissen des Tizian, Raffael und der Sofonisba Anguissola. Die hier so demonstrativ vor Augen geführten Kontraste - zwischen Künstlern und Künstlerinnen, zwischen jung (Raffael) und sehr alt Verstorbenen (Tizian, Sofonisba) -, insbesondere aber auch die ganz exzeptionelle, apokryphe (möglicherweise erst von van Dyck dazu gemachte) Darstellung des greisen Tizian mit seiner (schwangeren) Geliebten lassen sich nur verstehen, wenn man die , biologischen ' Produktionsmodelle der Frühen Neuzeit rekonstruiert und in ihrem Erklärungswert für die Zeitgenossen ernst nimmt: Sexuelle Prokreativität und künstlerische Kreativität wurden seit dem ausgehenden 15. Jahrhundert wieder zunehmend in Parallele gesetzt und lieferten gegenseitige Erklärungsmodelle. Dabei spielten Alterungsprozesse auf ganz unterschiedlichen Ebenen eine zentrale Rolle. Diskutiert wurden unterschiedliche Vorstellungen und Modelle der Frühbegabung, des geistig-künstlerischen Höhepunktes zu einem mittleren Lebensalter und der Vollendung als Greis. Diskutiert wurde auch das Verhältnis von körperlichem Verfall und unzerstörbarer , geistiger Jugend', von demonstrativem Überfluss und Einsatz der Liebeskräfte durch Künstler und Literaten oder aber von deren angeblich notwendiger Sublimierung. Vor diesem Hintergrund erscheint Tizian in der Inszenierung van Dycks - zwar nicht authentisch, aber wohl sehr im Sinne Tizians - als unerschöpflich potenter und produktiver KünstlerLiebhaber, wogegen der, verbrauchte" weibliche Körper der Sofonisba Anguissola schon lange nichts mehr, gebären ' kann. Die hier untersuchte Tradition des 16. und frühen 17. Jahrhunderts erweist sich so als zentral für eine ganze Reihe späterer, teils bis heute wirksamer Leitvorstellungen über Kreativität, herausragendes (männliches) Künstlertum und Alter.

Dies gilt im Übrigen auch für die beiden anderen, bislang nicht erwähnten $\mathrm{He}-$ roen der italienischen Hochrenaissance, Leonardo und Michelangelo. Nachdem das

sua illa Venere tenuisse visus est, qui cum omnia esse in imitatione statueret, maluit quod in aliis essent absolutius quam sua ipsius contemplari."

${ }^{40}$ Speroni, 1740, hier Bd. 1, 31 und 288. - S. auch Suthor, 2004, 21-27.

${ }^{41}$ Gigli, 1996. 
Leben Leonardos praktisch keine Nachricht über Frauen zu bieten hatte, zeigte wenigstens ein Gemälde aus der Leonardo-Nachfolge eine nackte Mona Lisa, die das Medaillonbildnis ihres Geliebten (möglicherweise erst im 18. Jahrhundert in dieser Form übermalt) an die Brust hält: eben das Porträt des greisen Leonardo. ${ }^{42}$ Der alte Michelangelo dagegen präsentierte sein künstlerisches Arbeiten, das überraschend im hohen Alter wieder der Skulptur galt, als eine Art Liebesbeweis gegenüber Gott, als Demut und selbstaufopfernde Arbeit. ${ }^{43}$ Wie unterschiedlich diese kreativen Liebes-Konzepte auch alle waren: Ganz unvorstellbar für die Konzeption der Frühen Neuzeit wäre gewesen, dass diese Ausnahme-Künstler mit ihren theoretischen und also zeitlos ,schönen Geistern“ im Alter überhaupt nicht mehr hätten produzieren können.

\section{Literaturverzeichnis}

Ahmed E (2008) Speaking the Unspeakable: Sterility and Infidelity in the Correspondance of Marguerite de Navarre and Guillaume Briçonnet. In: Leushuis R \& Zalloua ZA (Hrsg) Esprit généreux, esprit pantagruélicque. Essays by his students in honor of François Rigolot, Droz, Genf, S 95-111

Aretino P (1957-1960) Lettere sull'arte. Hrsg von E Camesasca. 3 Bde Ed. del Mil, Mailand

Artemieva I (2008) Die Venus vor dem Spiegel Barbarigo und der Dialogo della pittura von Ludovico Dolce. In: Ferino-Pagden S (Hrsg) Der späte Tizian und die Sinnlichkeit der Malerei, Kunsthistorisches Museum, Wien, S 225-231

Bake K (2005) Geschlechtsspezifisches Altern in einem Lebensalter-Zyklus von Tobias Stimmer und Johann Fischart. In: Hartung H (Hrsg) Alter und Geschlecht. Repräsentationen, Geschichte und Theorien des Alter(n)s, Transkript-Verlag, Bielefeld, S 113-133

Biegel G (Hrsg) (1993) Geschichte des Alters und ihre Zeugnisse von der Antike bis zur Gegenwart, Meyer, Braunschweig

Blake Smith A (1967) Anchora Inparo. In: Art Quarterly, 30:118-125

Bolzoni L (1984) Il teatro della memoria. Studi su Giulio Camillo, Liviana Ed., Padua

Borggrefe H (2006) Tizians ruhende Göttinnen und Dienerinnen der Liebe. In: Tacke A (Hrsg) ,... wir wollen der Liebe Raum geben“. Konkubinate geistlicher und weltlicher Fürsten um 1500, Wallstein-Verlag, Göttingen, S 393-421

Borghini R (1967) Il Riposo in cui della pittura e della scultura si favella. Hrsg von M Rosci, Ed. labor, Mailand

Breitenberg M (1996) Anxious Masculinity in Early Modern England, Cambridge Univ. Press, Cambridge

Brunetti M (1935) Una figlia sconosciuta di Tiziano. In: Rivista di Venezia, 14:175--184

Campbell E (1998) Old-Age Style and the Resistance of Practice in Cinquecento Art Theory and Criticism. Ph.D. University of Toronto

Chiari MA (1982) Incisioni da Tiziano. Catalogo del fondo grafico a stampa del Museo Correr. Venedig

Christadler M (2000) Kreativität und Geschlecht. Giorgio Vasaris ,Vite‘ und Sofonisba Anguissolas Selbst-Bilder, Reimer, Berlin

Clement R (1960) Picta Poesis. Literary and humanistic theory in Renaissance emblem books. Ed. di Storia e Letteratura, Rom

\footnotetext{
${ }^{42}$ Das Bildnis basiert auf dem erstmals 1589 publizierten Holzschnitt in der hier bereits Anm. 34 zitierten Icones-Ausgabe von Reusner; vgl. Pedretti, 1996, $127 \mathrm{f}$.

${ }^{43}$ Dazu nur Lavin, 1977/78, und Nagel, 2000.
} 
Courtright N (1996) Origins and Meanings of Rembrandt's Late Drawing Style. Art Bulletin $78: 485-510$

Cropper E (1971) Bound Theory and Blind Practice: Pietro Testa's notes on painting and the Liceo della Pittura. Journal of the Warburg and Courtauld Institutes, 34:262-296

Depauw C \& Luijten G (1999) Antoon van Dyck en de prentkunst, Antwerpen Open, Antwerpen/Amsterdam

De Vito G (1996-1997) Variazioni sul nome del Maestro dell'Annuncio ai pastori. In: Ricerche sul '600 napoletano, S 7-57

Donati A (2008) Giovanni Dò e i temi sapienziali. In: Ricerche sul '600 napoletano, S 57-69

Dormandy T (2000) Old Masters. Great Artists in Old Age, Hambledon Press, London/New York

Eikelmann R (Hrsg) (2006) Conrat Meit. Bildhauer der Renaissance, Hirmer, München

Emison P (2004) Creating the 'Divine' Artist from Dante to Michelangelo, Brill, Leiden/Boston

Filipczak ZZ (1997) Hot Dry Men, Cold Wet Women. The theory of humors in Western European art 1575-1700, American Federation of Arts, New York

Fletcher J (2003) Titian as a Painter of Portraits. In: Hope Ch et al. Titian, National Gallery, London S $29-42$

Fontanella G (1994) Ode. Hrsg von R Contarino. [San Mauro Torinese]

Freedman L (1995) Titian's Portraits Through Aretino's Lens, Penn State Univ. Press, University Park (PE)

Fusenig T (1997) Liebe, Laster und Gelächter. Komödienhafte Bilder in der italienischen Malerei im ersten Drittel des 16. Jahrhunderts, Romanistischer Verlag, Bonn

Garrard MD (1986) Artemisia Gentileschi. The image of the female hero in Italian Baroque art, Princeton University, Princeton (NJ)

Giarda C (1628) Bibliotheca Alexandrinae icones symbolicae ..., Bidelli, Mailand

Gigli GC (1996) La Pittura trionfante. Hrsg von B Agosti \& S Ginzburg. Porretta Terme

Graziani GC (1597) Di Orlando santo vita e morte. Treviso

Gregori M et al. (1994) Sofonisba Anguissola e le sue sorelle, Leonardo Arte, Cremona

Groos U (1998) Ars Musica in Venedig im 16. Jahrhundert, Olms, Hildesheim u. a.

Hollstein FWH (1951) Dutch and Flemish Etchings Engravings and Woodcuts ca. 1450-1700, Bd 4, Herzberger, Amsterdam

Hope C (1980) Titian, Jupiter, London

Hope C (1993) The Early Biographies of Titian. In: Manca J (Hrsg) Titian 500 [Studies in the History of Art 45], National Gallery, Washington, S 167-197

Jacobs F (1994) Woman's Capacity to Create. The unusual case of Sofonisba Anguissola. Renaissance Quarterly 47:74-101

Jacobs F (1997) Defining the Renaissance virtuosa. Women artists and the language of art history and criticism, Cambridge Univ. Press, Cambridge

Jaffé D (2001) New Thoughts on Van Dyck's Italian Sketchbook. Burlington Magazine, 143:614 624

Laam KP (2006) Aging the Lover: The Posies of George Gascoigne. In: Campbell E (Hrsg) Growing Old in Early Modern Europe. Cultural representations, Ashgate, Aldershot u. a., S 75-91

Lavin I (1977/78) The sculptor's 'last will and testament'. Bulletin. Allen Memorial Art Museum, 35:4-39

Loh MH (2007) Titian Remade. Repetition and Transformation of Early Modern Italian Art, Getty Center, Los Angeles

Lomazzo GP (1973) Scritti sulle arti, 2 Bde. Hrsg von RP Ciardi, Marchi \& Bertolli, Florenz

Long KP (Hgrs) (2002) High Anxiety. Masculinity in Crisis in Early Modern France, Truman State Univ. Press, Kirksville

Mancini M (1998) Tiziano e le corti d'Asburgo, Istituto Venete di Scienze, Lettere ed Arti, Venedig

Mauquoy-Hendrickx M (1991) L'Iconographie d'Antoine van Dyck, 2 Bde, Bibliothèque Royale, Brüssel

Nagel A (2000) Michelangelo and the Reform of Art, Cambridge Univ. Press, Cambridge 
Opmeerus P \& Beyerlinck L (1611) Opus Chronographicum Orbis Universi a Mundi Exordio usque ad annum M.DC.XI. Continens Historiam, Icones, et Elogia, Summorum Pontificum, Imperatorum, Regum, ac Virorum Illustrium; in duos tomos divisum. Antwerpen

Pallucchini R \& Rossi F (1983) Giovanni Cariani, Credito Bergamasco, Bergamo

Pardo M (1993) Artifice as Sedution in Titian. In: Turner J Grantham (Hrsg) Sexuality and Gender in Early Modern Europe, Cambridge Univ. Press, Cambridge, S 55-89

Partenio B (1565) De poetica imitatione. Venedig

Pedretti C (1996) Quella Puttana di Leonardo. Achademia Leonardi Vinci, 9:121-135

Perini G (2000) Una certa idea di Raffaello nel Seicento. In: Borea E \& Gasparri C (Hrsg) (2000) L'idea del bello. Viaggio per Roma nel Seicento con Giovan Pietro Bellori, Bd 1, DeLuca, Rom, S 153-161

Persio A (1999) Trattato dell'ingegno dell'huomo. Hrsg von L Artese. Ist. Ed. e Poligraf. Internazionali, Pisa/Rom

Pfisterer U (2003) Erste Werke und Autopoiesis. Der Topos künstlerischer Frühbegabung im 16. Jahrhundert. In: Pfisterer U \& Seidel M (Hrsg) Visuelle Topoi. Erfindung und tradiertes Wissen in den Künsten der Renaissance, Dt. Kunstverlag, München/Berlin, S 263-302

Pfisterer U (2011) Traurige Musen. Jacopo de' Barbari zu Malerei, Dichtung und Kulturtransfer im Norden. In: Müller M et al. (Hrsg) Kulturtransfer am Fürstenhof. [Im Erscheinen]

Pfisterer U \& Wimböck G (Hrsg) (2011) Novità - Neuheitskonzepte in den Bildkünsten um 1600, Diaphanes, Zürich/Berlin

Pierio Valeriano G (1567) Hieroglyphica, Basel

Puppi L (Hrsg) (2007) Tiziano. L'ultimo atto, Skira, Mailand

Raupp H-J (1993a) Der alte Künstler und das Alterswerk. In: Döring T et al. (Hrsg) Bilder vom alten Menschen in der niederländischen und deutschen Kunst 1550-1750, Herzog Anton-Ulrich Museum, Braunschweig, S 87-97

Raupp H-J (1993b) Johann Heintz: Die Malerei flieht den greisen Künstler. In: Döring T et al. (Hrsg) Bilder vom alten Menschen in der niederländischen und deutschen Kunst 1550-1750, Herzog Anton-Ulrich Museum, Braunschweig, S 305f (Kat 109)

Reinhardt S (1999) Tizian in England. Zur Kunstrezeption am Hof Karls I., Böhlau, Frankfurt a. M.

Reusner N (1589) Icones sive imagines vivae literis cl. Virorum, Basel

Ripa C (1645) Iconologia ... divisa in tre libri ... Venedig

Rösler A, Nesselhauf D, Pfisterer U et al. (2010) ,Old-Age Style' or ,Sick Style"? On the Artistic Development of Cognitively Healthy and Cognitively Impaired Artists with Advancing Age. Journal of Aging, Humanities and the Arts, 4:368-376

Rosand D (2009) Titian Draws Himself. Artibus \& Historiae, 30/59:65-71

Schütze S (2010) Teoria e pratica del disegno napoletano e l'arte del Cavalier Massimo. In: Solinas F \& Schütze S (Hrsg) Le dessin napoletain, DeLuca, Rom, S 81-90

Schuster Cordone C (2009) Le crépuscule du corps. Images de la viellesse féminine, Infolio, Gollion

Seipel W (Hrsg) (2001) Dipingere la musica. Musik in der Malerei des 16. und 17. Jahrhunderts, Skira, Wien/Mailand

Sohm P (2007) The Artist Grows Old. The Aging of Art and Artists in Italy, 1500-1800, Yale Univ. Press, New Haven/London

Soussloff CM (1987) Old Age and Old-Age Style in the ,Lives' of Artists: Gianlorenzo Bernini. Art Journal, 115-121

Speroni S (1740) Opere, 5 Bde. Venedig

Suthor N (2004) Augenlust bei Tizian. Zur Konzeption sensueller Malerei in der frühen Neuzeit, Fink, München

Tizianello (2009) Breve compendio della vita di Tiziano (1622). Hrsg von L Puppi. Edizioni il Polifilo, Mailand

Tongiorgi Tomasi L \& Tosi A (Hrsg) (2009) Il cannocchiale e il pennello. Nuova scienza e nuova arte nell'età di Galileo, Giunti, Florenz

Van Dyck A (1940) Italienisches Skizzenbuch. Hrsg von G Adriani. Schroll, Wien 
Van Mander K (1604) Het schilder-boek ... Haerlem

Verrier F (2003) Frammenti di un discorso sugli amori degli artisti nella prima edizione delle Vite. Letteratura \& Arte, 1:103-115

Wałek J (1991) The Czartoryski Portrait of a „Youth“ by Raphael. Artibus \& Historiae, 12/24:201224

Wanders H (1983) Das springende Böckchen - Zum Tierbild in den dekadischen Lebensalterdarstellungen. In: Joerißen P \& Will C (Hrsg) Die Lebenstreppe. Bilder der menschlichen Lebensalter. Rheinland-Verlag, Köln, S 61-71

Wethey H (1971) The Paintings of Titian. II. The Portraits, Phaidon, London

Wheelock AK Jr et al. (Hrsg) (1991) Van Dyck: Paintings, Thames and Hudson, Washington/Den Haag

Winner M (1992) „... una certa idea“: Maratta zitiert einen Brief Raffaels in einer Zeichnung. In: Winner M (Hrsg) Der Künstler über sich in seinem Werk, Akademie Verlag, Weinheim, S $511-570$ 